(C)1983. The Genetical Society of Great Britain

\title{
DIRECTIONAL SELECTION FOR BODY WEIGHT IN CONSTANT AND VARIABLE ENVIRONMENTS IN DROSOPHILA MELANOGASTER
}

\author{
C. M. KAMMERER ${ }^{1}$ AND S. S. Y. YOUNG ${ }^{2}$ \\ ${ }^{1}$ Department of Genetics, Southwest Foundation for Research and Education, \\ San Antonio, Texas, U.S.A. \\ ${ }^{2}$ Department of Genetics, The Ohio State University, Columbus, Ohio, U.S.A.
}

Received 16.vii.82

\section{SUMMARY}

\begin{abstract}
A comparison of the results of 13 generations of directional selection (with control lines) for higher body weight in Drosophila melanogaster in two variable temperature environments (cyclic and random) with those of three constant temperature environments $\left(19^{\circ}, 24.5^{\circ}\right.$, and $\left.27^{\circ} \mathrm{C}\right)$ is made. Rates of genetic gain obtained in the variable environments were similar to those achieved in constant temperature environments. The ability to buffer against environmental changes, in terms of body weight and some fitness characteristics, was altered by selection for higher body weight but not by the environmental regimes. Lines selected at the presumed optimum temperature $\left(24.5^{\circ} \mathrm{C}\right)$ performed best at all temperatures. These results indicate that long-term genetic gain in a quantitative trait through directional selection is probably unaffected in any major way by normal variations in environmental conditions and that selection for overall performance should be carried out in the most favourable environment.
\end{abstract}

\section{INTRODUCTION}

The response of a population to many cycles of selection in constant and in heterogeneous environments is a subject of longstanding interest. Populations experiencing more variable environmental conditions are thought to require relatively more genetic or phenotypic flexibility for survival in comparison with populations adapted to more stable habitats (Darlington, 1939; Mather, 1943; Thoday, 1953). Some evidence from natural and experimental populations of Drosophila support this premise (Beardmore, 1960; Tantawy and Mallah, 1961; Beardmore and Levine, 1963; Long, 1970; Band, 1972; Nevo, 1978), but the evidence is by no means extensive or uniform (Gottlieb and Sacks, 1968; Brown and Feldmeth, 1971). The ability of a population to buffer against changes in environmental conditions may be related to the genetic variability of the population (Fontdevila, 1973; MacDonald and Ayala, 1974), but the genetic mechanism involved in this possible association between genetic variability and environmental variability remains unclear, and may in fact differ for different traits (MacKay, 1981).

A few long-term selection experiments reported in the literature are relevant to this problem of predicting the effects of many cycles of selection on a population evolving through heterogeneous environments. These experiments were designed mainly to observe the response of a population under selection in a constant environment and then subsequently to test the product of selection in several different environments (Falconer, 1960; Druger, 1962; Hardin and Bell, 1967; Jinks and Connolly, 1973, 1975). In general, the results of these experiments showed that the greatest mean 
response over a variety of environments came from selection for performance in the least favourable environment. This general conclusion may have some practical implication because animals or plants are usually selected in one environment and then expected to thrive in many different environments. In addition, the above experiments all found that maximum genetic progress was made in the environment in which the progeny will be maintained. This conclusion is not surprising since genetic correlations between measurements of the same trait in different environments (Hazel, 1943) cannot be expected to exceed unity. For a correlated response to selection to be higher than a direct response, a large difference in heritability and a very high genetic correlation between measurements of the trait taken in different environments are necessary. Satisfying both of these conditions in any one case would be difficult.

Due to the absence of long-term selection experiments under variable environmental conditions, there is little information available on the relative effects of selection, in terms of both direct and indirect responses, between constant environments and varying environments. In the present study, experiments were carried out to investigate the effects of selection for a metric trait in three constant environments (Experiment I) and two variable environments (Experiment II). Temperature was used as the environmental variable and Drosophila body weight was used as the metric character under directional selection. In addition, the fecundity and fertility of individual flies were observed to assess the correlated response in fitness due to selection for higher body weight. Due to limitations of labour, experiment II was carried out subsequent to experiment I. For this reason, differences in the results of the two experiments may be influenced by the difference in time periods or by genetic sampling. However, we were careful to provide, as nearly as possible, identical experimental conditions for the two experiments.

\section{MATERIALS AND METHODS}

The genetic stock used in experiment I was synthesized by crossing two laboratory strains, "Crossbred" and "Texas" in 1976. "Crossbred" was originally synthesized from four-way crosses of four laboratory strains in 1971. "Texas" was founded from several Drosophila melangaster captured in 1974. This large synthetic stock was maintained by random mating for 12 generations prior to the commencement of the experiment. The genetic stock was resynthesized from "Texas" and "Crossbred" in experiment II.

All lines in this study were reared in half-pint milk bottles, each containing approximately $50 \mathrm{ml}$ of standard Drosophila cornmeal medium or, when indicated, reared in 10 dram glass vials with approximately $5 \mathrm{ml}$ of food in each vial. All flies used in the study were aged, sexes separate, two days before being individually weighed to the nearest one hundredth of a milligram on a Schultz model 30 microbalance. The flies of each generation of each line were reared in one of three temperatures: $19^{\circ}$, $24.5^{\circ}$, or $27^{\circ} \mathrm{C}$. The $19^{\circ}$ and $27^{\circ} \mathrm{C}$ temperatures were maintained by Precision Scientific constant temperature cabinets. The $24.5^{\circ} \mathrm{C}$ temperature was maintained in a controlled temperature room.

The experimental procedure was carried out in a similar fashion in both experiments I and II, except as noted. This procedure consisted of three 
steps:

\section{(i) Estimation of differential fertility and fecundity}

Differential fertility (the proportion of females producing one or more offspring) and fecundity (number of offspring per fertile female) were estimated at the beginning and end of this experiment in the following manner. Virgin male and female flies were collected and pair-mated in separate vials and then randomly placed in one of the three temperatures. Offspring from each vial were counted over a period equal to the generation length of the flies in the temperature in which they were raised, i.e., 8 days in $27^{\circ}, 9$ days in $24.5^{\circ}$, and 20 days in $19^{\circ} \mathrm{C}$. This procedure insured that no second generation flies were counted.

\section{(ii) Selection phase}

The base population of experiment I was partitioned into six experimental lines (three selected and three control) in the following manner. Forty pairs of parents were chosen at random from the base population. Six male and six female progeny from each pair of parents were randomly assigned to one of six lines that were designated HS, HC, WS, WC, CS, CC. Hence, each line originated from 40 virgin females and 40 males. Also, the gene pools of all six lines were similar. The 40 pairs of parents within each line were randomly assigned, 10 pairs per bottle, to one of four bottles.

In experiment II, 30 pairs of parents were randomly chosen from the base population. Eight male and eight female progeny from each pair of parents were randomly assigned to one of eight lines that were designated $\mathrm{RC} 1, \mathrm{RC} 2, \mathrm{R} 1, \mathrm{R} 2, \mathrm{SC} 1, \mathrm{SC} 2, \mathrm{~S} 1, \mathrm{~S} 2$. Thus, the gene pools of the four selected lines and four control lines were similar. The 30 pairs of parents within each line were randomly assigned, 10 pairs per bottle, to one of three bottles. The two control lines in each variable environment were combined to form one line (RC or SC) because only one control line per variable environment was needed. In addition, each pooled control line, with its greater number of parents $(60)$, was expected to provide more accurate information on the selected lines.

After being reared in the bottles for six days, all adults in all lines in both experiments were removed.

In the constant environment experiment, all generations of the $\mathrm{H}$ lines were raised at $27^{\circ} \mathrm{C}$, all generations of the $\mathrm{W}$ lines were raised at $24.5^{\circ} \mathrm{C}$, and all generations of the $\mathrm{C}$ lines were raised at $19^{\circ} \mathrm{C}$. There were two lines for each temperature, a selection line (S) and a control line (C).

Two variable temperature environments were studied in experiment II. The environments were made to vary from generation to generation. Under the cyclic (S) regime, the parents were placed in one temperature environment and their progeny were reared, aged, and weighed in the same environment. The 30 or 60 pairs of progeny chosen to be the parents of the next generation were placed in a different environment and their resultant progeny raised in this new environment. The choice of the temperature environment was dictated by the following prearranged temperature cycle. 


$\begin{array}{lccccccccccccc}\text { Environment } & \text { H } & \text { W } & \text { C } & \text { H } & \text { W } & \text { C } & \text { H } & \text { W } & \text { C } & \text { H } & \text { W } & \text { C } & \text { H } \\ \text { Generation } & 1 & 2 & 3 & 4 & 5 & 6 & 7 & 8 & 9 & 10 & 11 & 12 & 13\end{array}$

where $\mathrm{H}=27^{\circ} \mathrm{C}, \mathrm{W}=24 \cdot 5^{\circ} \mathrm{C}$, and $\mathrm{C}=19^{\circ} \mathrm{C}$. Every three generations the populations underwent a complete temperature cyele. There were two replicates of the cyclic selection line (S1, S2) and one control line (SC). The replicates were selected separately, but S1, S2, and SC were run simultaneously.

The random environmental regime also had a control line (RC) and two selection line replicates $(\mathrm{R} 1, \mathrm{R} 2)$. the experimental procedure was identical to that of the cyclic lines with the exception that each generation was transferred to a different environment according to the following predetermined schedule.

$\begin{array}{lccccccccccccc}\text { Environment } & \text { W } & \text { H } & \text { C } & \text { W } & \text { C } & \text { H } & \text { C } & \text { W } & \text { H } & \text { W } & \text { H } & \text { C } & \text { W } \\ \text { Generation } & 1 & 2 & 3 & 4 & 5 & 6 & 7 & 8 & 9 & 10 & 11 & 12 & 13\end{array}$

In the control line of experiment I (Experiment II), 40 (60) pairs of flies were collected, aged (sexes separate), and weighed. These flies were then randomly assigned, 10 pairs per bottle, to one of four (six) bottles to continue the control line. This procedure was followed for 13 generations.

Approximately 160-200 (150) pairs of flies for each selection line were collected per generation, aged (sexes separate), and weighed. The heaviest 40 (30) pairs were randomly assigned, 10 pairs per bottle, to one of four (three) bottles to continue the selection line. The selection intensity for each generation was thus approximately $20-25$ per cent (20 per cent). Selection was practiced for 12 generations.

\section{(iii) Testing phase}

At generation 13, 90 pairs of flies from each line were collected, aged, weighed, and randomly assigned (10 pairs per bottle) to one of nine bottles (for a total of 54 bottles over all lines in each experiment). Three bottles per line were then placed in each of the three experimental temperatures, $27^{\circ}, 24 \cdot 5^{\circ}$, and $19^{\circ} \mathrm{C}$. Twenty-seven pairs of flies per bottle were collected, aged, and weighed. In experiment I, an estimate of average net fitness was obtained by dividing the total number of offspring that emerged from each bottle by 10 . In addition, estimates of individual fertility and fecundity were made in the temperature environment in which the line had been maintained. In experiment II, individual fertility and fecundity counts were made in each of the three experimental temperatures for each of the six lines.

The following mixed model was used in the ANOVA of body weight of each sex in experiment I:

$$
Y_{i j k l m}=\mu+S_{i}+E_{j}+T_{k}+S E_{i j}+S T_{i k}+E T_{j k}+S E T_{i j k}+B_{l(i j k)}+\varepsilon_{i j k l m}
$$

where $Y_{i j k l m}$ is the individual body weight of the $m$ th fly $(m=1, \ldots, 27)$, in the $l$ th bottle $(l=1, \ldots, 3)$, the $k$ th testing temperature $(k=1, \ldots, 3)$, the $j$ th experimental temperature $(j=1, \ldots, 3)$, and the $i$ th line $(i=$ 1,2 -selected or control). 
ANOVA of body weight of each sex in experiment II was done using:

$$
Y_{k r s l m}=\mu+T_{k}+P_{r}+T P_{k r}+L_{s(r)}+T L_{k s(r)}+B_{l(k r s)}+\varepsilon_{k r s l m}
$$

where $Y_{\text {krslm }}$ is the body weight of the $m$ th fly $(m=1, \ldots, 27)$, in the $l$ th bottle $(l=1, \ldots, 3)$, the $s$ th line $(s=1, \ldots, 3)$, the $r$ th pattern $(r=$ $1, \ldots, 2)$, and the $k$ th testing temperature $(k=1, \ldots, 3)$. Individuals and bottle effects were assumed to be random variables while lines, pattern, experimental temperatures, and testing temperature were assumed to be fixed. Similar ANOVA's were carried out on the fertility, fecundity, and net fitness data.

Individual body weight, fecundity, and net fitness data were transformed to logarithms for analysis.

\section{RESULTS}

The effects of temperature on body weight and some fitness characteristics were measured on the base populations (table 1). Unfortunately, in experiment II, samples from the base population reared at $19^{\circ} \mathrm{C}$ were lost prior to weighing. Body weight and generation length decreased in both

TABLE 1

Effects of temperature on the base population in experiment I (experiment II)

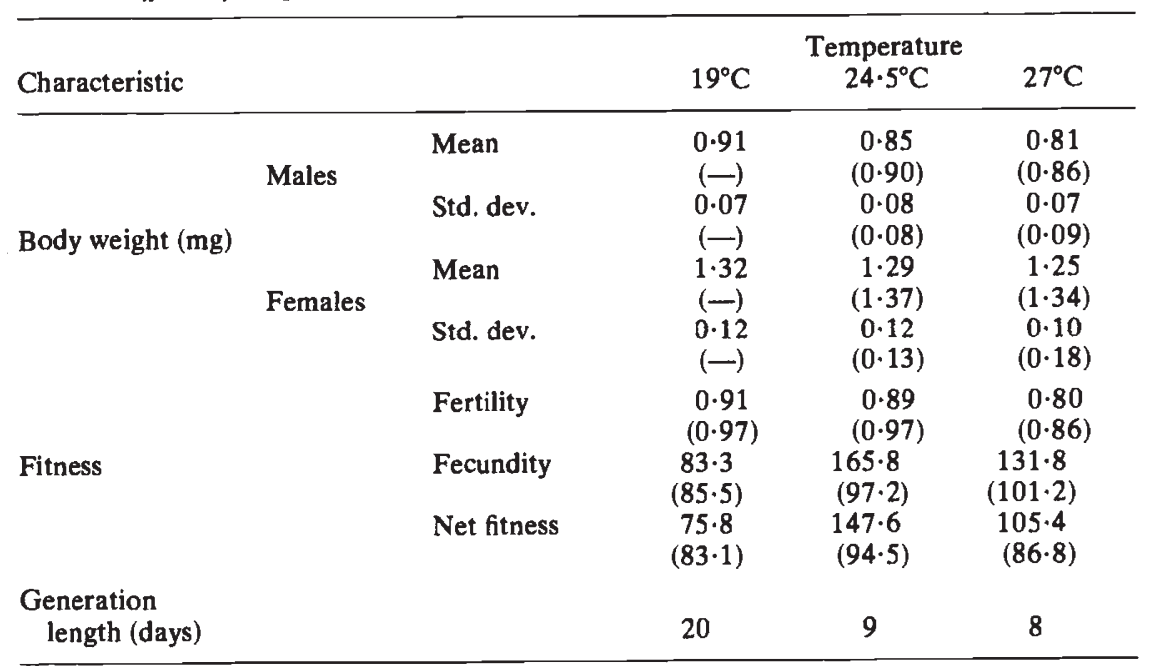

males and females with increasing temperature. Although net fitness was highest at $24.5^{\circ} \mathrm{C}$ and lowest in $19^{\circ} \mathrm{C}$, fertility, fecundity, and net fitness in different temperatures were not significantly different. Heritability in the base population of experiment I (experiment II) was estimated by means of a full sib ANOVA (Turner and Young, 1979) and gave values of $0.2(0.73)$ with a standard error of $0.3(1.23)$.

The mean weights for the three constant environment control lines fluctuated randomly over the generations (fig. 1). In the cyclic control (SC) line, the mean body weight varied randomly until generation seven, after 

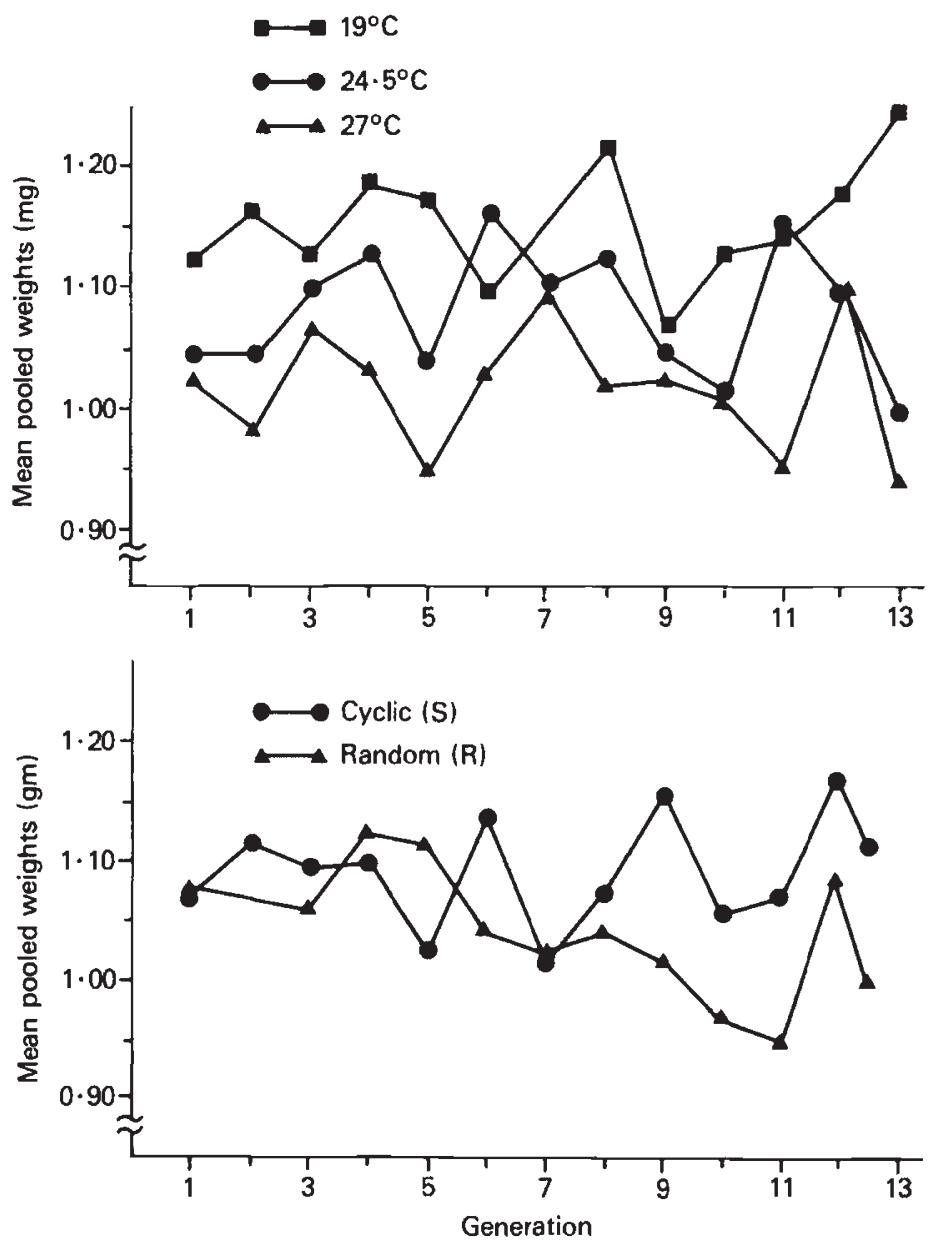

Fig. 1. Mean pooled weights (male and female) of the control lines across generations in constant environments (top) and variable environments (bottom).

which the mean body weights increased or decreased in a cyclic pattern following that of the temperature environment. The regression coefficients were not significantly different from zero. The random environment control line ( $R C)$, however, did have a significant negative slope due to the decrease in mean body weight from generation six onward (fig. 1).

Selection responses within constant and variable environment regimes (fig. 2) were compared using realised heritabilities. The estimates of heritability in each selection line [with standard error (Hill, 1972)] were as follows: (a) constant environments: $27^{\circ}, 0.20(0.01) ; 24.5^{\circ}, 0.13(0.01)$; $27^{\circ}, 0.17(0.01) ;$ (b) variable environments: S1, $0.16(0.01)$; $S 2$, $0.15(0.01) ; \mathrm{R} 1,0.18(0.01) ; \mathrm{R} 2,0.22(0.01)$. The $24.5^{\circ} \mathrm{C}$ line had a significantly lower rate of genetic gain than either the $19^{\circ} \mathrm{C}$ or the $27^{\circ} \mathrm{C}$ line. In experiment II, estimates of realised heritability were not significantly different between replicates in the cyclic environment, but significant differences were found between replicates in the random environment. 

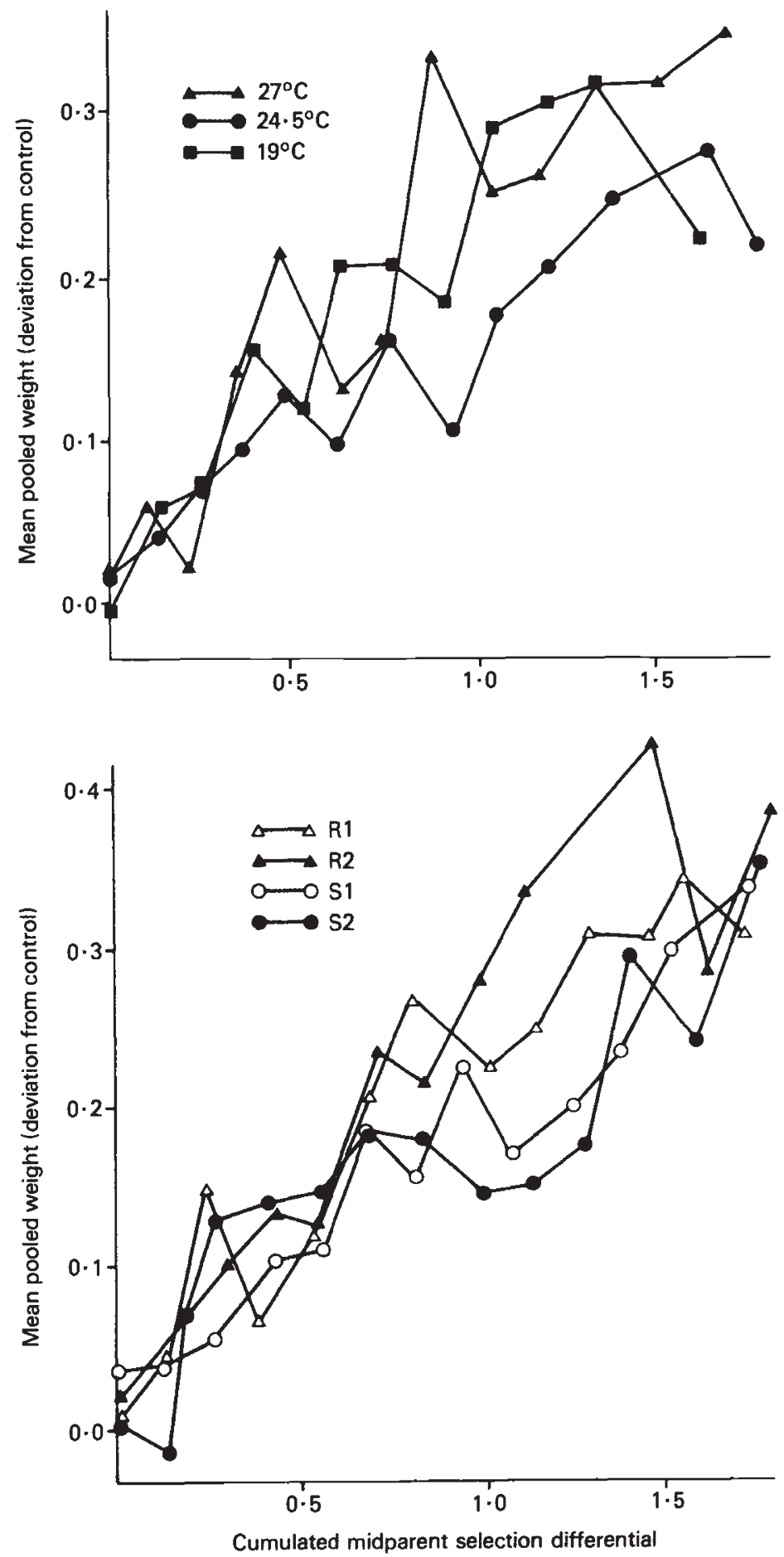

FIG. 2. Rates of genetic gain in constant environments (top and variable environments (bottom). 
Offspring from all experimental lines were raised in each of the three different temperature environments in the testing phase. Thus, estimates of genetic correlation, genotype $\times$ environment interaction, and buffering ability (for body weight and net fitness) obtained from flies selected under different regimes could be compared.

\section{(i) Genetic correlation}

Two kinds of realised genetic correlation, the average realised genetic correlation, $r_{A G}$, and the individual realised genetic correlation $r_{G}$, were calculated from the testing phase data in experiment $I$ as described below (Falconer, 1960a).

$$
r_{A G}=\left(C R_{X} C R_{Y} / R_{X} R_{Y}\right)^{1 / 2} \text { and } r_{G}=C R_{X} i_{X} h_{X} / R_{X} i_{Y} h_{Y}
$$

where

$$
\begin{aligned}
C R_{X}= & \text { correlated response at temperature } X \text { resulting from } \\
& \text { selection in } Y ; \\
R_{X}= & \text { direct response at temperature } X \text { due to selection in } X ; \\
i= & \text { cumulative selection differential; } \\
h= & \text { the square root of the heritability. }
\end{aligned}
$$

The realised genetic correlations for body weight between temperatures were generally high. The average genetic correlation $\left(r_{A G}\right)$ decreased with increasing difference in temperatures, e.g., $r_{\left(27^{\circ}, 24.5^{\circ}\right)}=0.96, r_{\left\{24.5^{\circ}, 19^{\circ}\right)}=$ $0.84, r_{\left(27^{\circ}, 19^{\circ}\right)}=0.69$. Estimates of the individual genetic correlations, $r_{G}$ (table 2), also showed that the correlated responses between temperature were asymmetrical, e.g., flies selected in the higher temperature showed a highly correlated gain when raised in the cold environment, while flies selected in the cold temperatures performed poorly in the high temperature.

TABLE 2

Realised genetic correlations $\left(\mathrm{r}_{\mathrm{G}}\right)$ for body weight

\begin{tabular}{cccc}
\hline $\begin{array}{c}\text { Selection } \\
\text { temperature }\end{array}$ & $19^{\circ}$ & $\begin{array}{c}\text { Testing temperature } \\
24.5^{\circ}\end{array}$ & $27^{\circ}$ \\
\hline $19^{\circ}$ & - & 0.53 & 0.27 \\
$24.5^{\circ}$ & 0.91 & - & 1.01 \\
$27^{\circ}$ & 0.86 & 0.85 & - \\
\hline
\end{tabular}

This asymmetry of correlated responses is in agreement with earlier findings reported by Falconer and Latyszewski (1952) and Falconer (1960) in mice and Druger (1962) and Anderson (1966) in Drosophila.

\section{(ii) Genotype $\times$ environment interactions}

After 13 generations of selection, progeny of each line were tested in the three temperatures (table 3 ). The sources of variation in an ANOVA of body weight due to testing temperature and selection in experiment $I$, 
TABLE 3

Testing phase body weight means $(\mathrm{mg})$ of control $(C)$ and selected $(S)$ lines

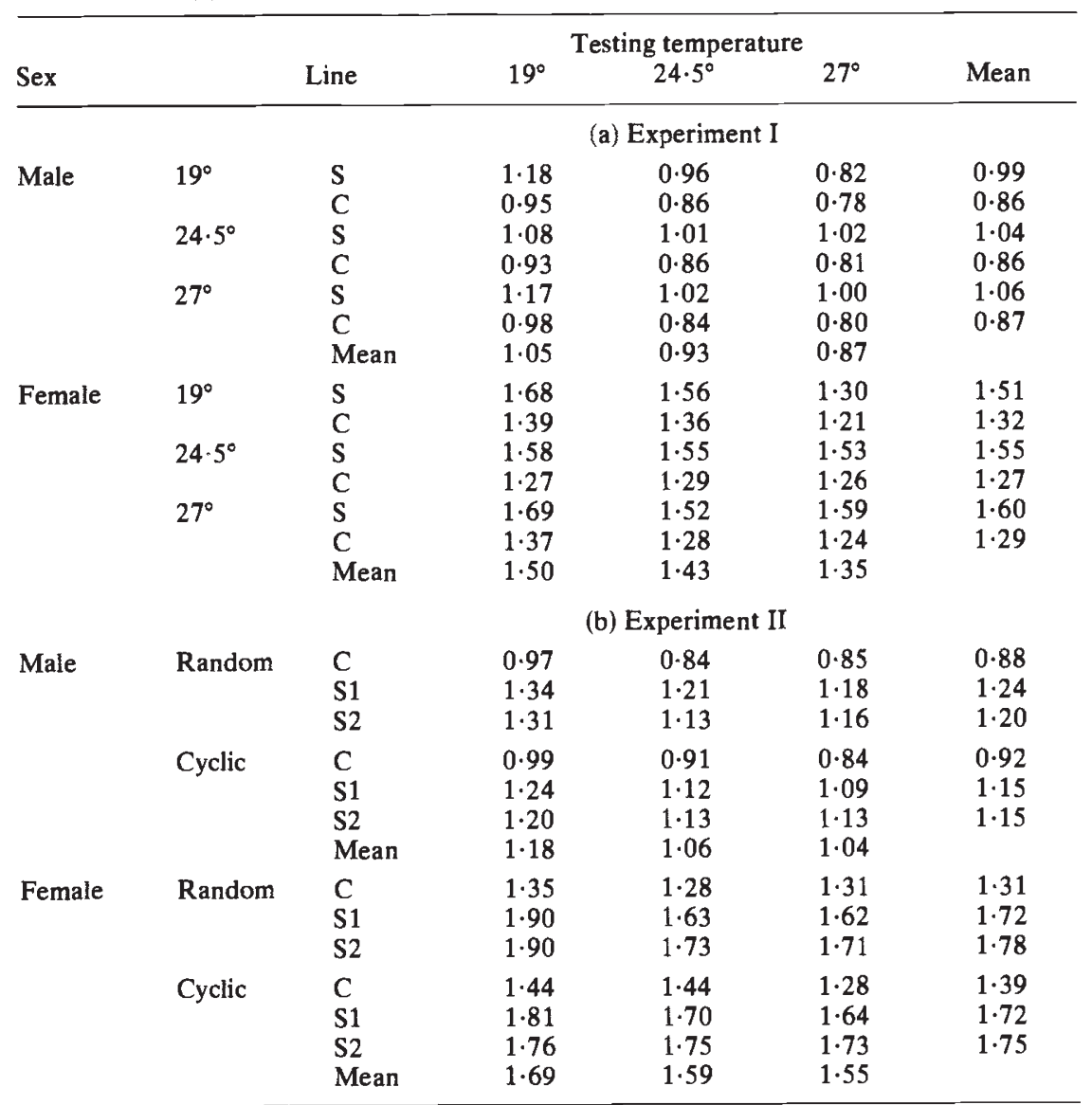

and testing temperature and lines in experiment II, were highly significant for both sexes, i.e., selected lines had higher mean weights than control lines and mean weight also increased with decreasing testing temperature. These two sources represented 53-63 per cent and 68-78 per cent of the total phenotypic variation in experiments I and II, respectively. Of special interest was the presence of a highly significant $(p<0.001)$ genotype by environment interaction that implied flies evolved in different temperature regimes performed differently in the testing temperatures. This interaction was foreshadowed by the genetic correlations presented earlier (details of ANOVA not shown).

Relative sensitivities of selected and unselected flies to environmental changes were compared using separate ANOVA of body weight data of control and selected lines (table 4). As can be seen there are highly significant genotype by environment effects for flies selected in different temperature regimes but the same effects were negligible for unselected flies. Further investigation of the genotype $\times$ environment interaction constants in experiment I revealed that each selected line performed well in 
TABLE 4

Analysis of variance of body weight $[\ln (\mathrm{mg})]$ of control and selected lines

\begin{tabular}{|c|c|c|c|c|c|c|}
\hline \multirow[b]{3}{*}{ Source } & \multirow[b]{3}{*}{ df } & \multicolumn{5}{|c|}{ Mean square } \\
\hline & & \multicolumn{2}{|c|}{ Control } & \multirow[b]{2}{*}{ df } & \multicolumn{2}{|c|}{ Selected } \\
\hline & & Males & Females & & Males & Females \\
\hline & \multicolumn{6}{|c|}{ (a) Experiment I } \\
\hline $\begin{array}{l}\text { Experimental } \\
\text { temperatures }(E)\end{array}$ & 2 & $0 \cdot 008$ & $0 \cdot 120$ & 2 & $0.353^{*}$ & 0.474 \\
\hline \multicolumn{7}{|l|}{ Testing } \\
\hline temperatures $(\mathrm{T})$ & 2 & $1 \cdot 560^{* * *}$ & $0.742^{*}$ & 2 & $2 \cdot 670^{* * *}$ & $1.984^{* * *}$ \\
\hline ET & 4 & 0.038 & $0 \cdot 204$ & 4 & $0.483^{* *}$ & $0.900^{* * *}$ \\
\hline \multicolumn{7}{|l|}{ Bottles within } \\
\hline \multirow[t]{2}{*}{ Error } & 702 & $0 \cdot 006$ & $0 \cdot 017$ & 702 & $0 \cdot 007$ & 0.024 \\
\hline & \multicolumn{6}{|c|}{ (b) Experiment II } \\
\hline $\begin{array}{l}\text { Testing } \\
\text { temperatures }(\mathrm{T})\end{array}$ & 2 & $0 \cdot 821^{* * *}$ & $0.434^{*}$ & 2 & $1 \cdot 796^{* * *}$ & $2.434^{* * *}$ \\
\hline Patterns (P) & 1 & $0 \cdot 129$ & $0.690^{*}$ & 1 & $1 \cdot 227^{* *}$ & $0 \cdot 081$ \\
\hline TP & 2 & 0.065 & $0.366^{*}$ & 2 & $0.092^{*}$ & $0.697^{* *}$ \\
\hline Replicates (R) & - & & & 2 & $0 \cdot 109^{*}$ & $0 \cdot 317^{*}$ \\
\hline TR & - & & & 4 & 0.057 & $0 \cdot 153$ \\
\hline $\begin{array}{l}\text { Bottles within } \\
\text { treatments }\end{array}$ & 12 & $0.039 * * *$ & $0.094 * * *$ & 24 & $0 \cdot 025^{* * *}$ & $0 \cdot 061^{* * *}$ \\
\hline Error & 468 & 0.004 & 0.015 & 936 & 0.009 & 0.023 \\
\hline
\end{tabular}

${ }^{*} P<0.05$.

** $P<0.01$.

*** $P<0 \cdot 001$.

the temperature in which it was maintained (details not shown). This result is in accordance with the literature cited earlier.

\section{(iii) Net fitness}

Effects of selection in different environmental regimes on fertility, mean fecundity, and mean net fitness were investigated. In experiment I, measurements were made on the base population and, after 13 generations, on all the control and selection lines (table 5). Lines raised at $24 \cdot 5^{\circ} \mathrm{C}$ were superior

\section{TABLE 5}

Mean fecundity, fertility, and net fitness of the base population and the 13th generation of all selected and control lines in experiment $I$

\begin{tabular}{|c|c|c|c|c|c|c|c|c|c|}
\hline \multirow[b]{2}{*}{ Line } & \multicolumn{3}{|c|}{ Fertility } & \multicolumn{3}{|c|}{ Fecundity } & \multicolumn{3}{|c|}{ Net fitness* } \\
\hline & $19^{\circ}$ & $24 \cdot 5^{\circ}$ & $27^{\circ}$ & $19^{\circ}$ & $24 \cdot 5^{\circ}$ & $27^{\circ}$ & $19^{\circ}$ & $24 \cdot 5^{\circ}$ & $27^{\circ}$ \\
\hline \multicolumn{10}{|l|}{ Base } \\
\hline population & 0.91 & 0.89 & $0 \cdot 80$ & 82.9 & $166 \cdot 7$ & $131 \cdot 8$ & $75 \cdot 8$ & $147 \cdot 6$ & $105 \cdot 4$ \\
\hline Control & 0.86 & $1 \cdot 00$ & 0.80 & $90 \cdot 3$ & $159 \cdot 6$ & $113 \cdot 0$ & $77 \cdot 4$ & $159 \cdot 6$ & 90.4 \\
\hline Selected & 0.89 & $1 \cdot 00$ & 0.89 & $75 \cdot 8$ & $113 \cdot 4$ & $93 \cdot 1$ & $67 \cdot 1$ & $113 \cdot 4$ & $82 \cdot 5$ \\
\hline
\end{tabular}

* Net fitness $=$ fertility $\times$ fecundity. 
to the lines raised at $27^{\circ}$ or $19^{\circ} \mathrm{C}$ both before and after selection in terms of fertility, fecundity, and net fitness. The differences in fecundity and net fitness are statistically significant. Selection had a significant effect on fecundity, but not on fertility or net fitness. These results suggested that $24 \cdot 5^{\circ} \mathrm{C}$ was the optimum temperature and that selection lowered the overall fitness of the flies.

Average net fitness means obtained from total bottle counts in the three testing temperatures are shown in table 6 . No significant genotype by environment interaction was found.

TABLE 6

Testing phase means of finess characteristics

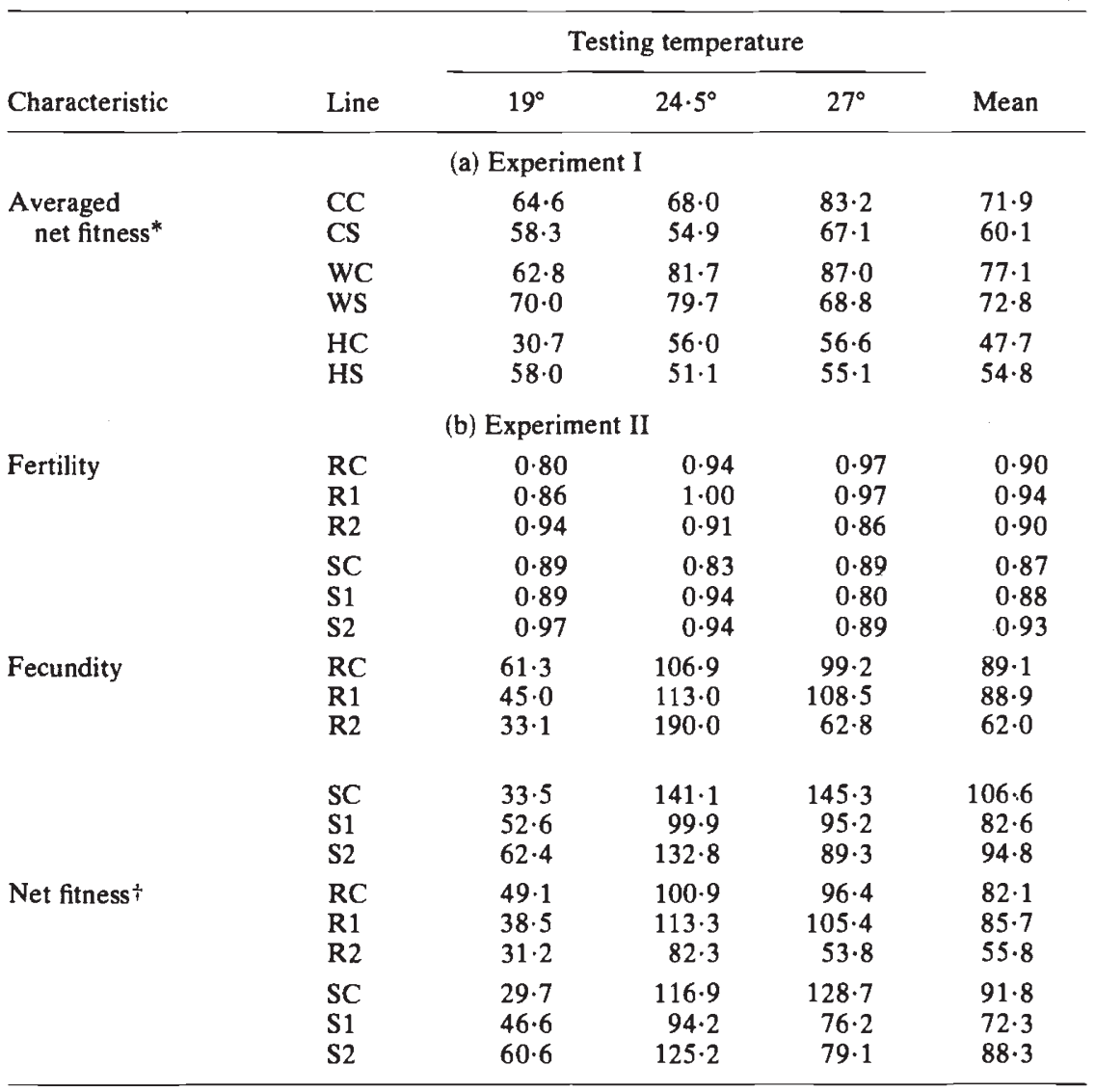

* Bottle means.

+ Vial means.

In experiment II, fertility was unaffected by either temperature or environmental regime. Different testing temperatures had significantly different effects on fecundity in the selected lines and on net fitness of both control and selected populations. There was also a significant temperature by pattern interaction effect on net fitness of the selected populations. 


\section{(iv) Buffering ability}

Comparison of the relative sensitivity of the selected lines to changes in environmental conditions is also of interest. One simple measure of comparison can be obtained by calculating within-line variances over testing temperatures of the means of each trait (details not presented here). A relatively low variance for a line is an indication of its greater uniformity of performance over testing temperatures and, thus, is an index of its buffering ability. The buffering indexes of any two lines were compared by the usual variance ratio test, but the test is insensitive due to the small number of degrees of freedom associated with each variance. Nevertheless, selection did have some effect on the buffering ability of lines in terms of both body weight and net fitness. Among the selected lines, the $24.5^{\circ} \mathrm{C}$ lines exhibited much smaller variation than did the $27^{\circ} \mathrm{C}$ and $19^{\circ} \mathrm{C}$ lines for body weight. However, no significant differences were observed between any lines for body weight or net fitness.

In experiment II, female body weight means of cyclic selection line two (S2) were significantly more uniform across testing temperatures than those of its replicate (S1) and the cyclic control line (SC). Buffering ability within the random lines did not differ significantly for body weight. Cyclic environment lines were less variable than random environment lines in terms of body weight but this difference was not significant. No significant differences were found between any of the lines in terms of net fitness.

Arguments could be made that some of the differences in the above responses were due to culture density rather than body weight differences, since body weight increased with decreasing fecundity in the base populations (table 1). However, inspection of tables 3 and 6 reveals that the highest fecundity is not necessarily concomitant with the highest body weight, or vice versa, within lines across temperatures or across lines within temperatures. Also, previous work in our lab using the above methodology indicated that culture densities probably would have negligible effects on our results.

\section{Discussion}

The present experimental design attempts to mimic, in a mechanical way, selection in constant and variable environments. The environmental variable used is a powerful agent which can drastically alter both the body weight, fertility, and fecundity of flies.

Before comparing results obtained from the two experiments, some of the expectations from earlier studies will be recalled. Selection in the variable environments was expected to be less efficient. Also, flies selected under variable environmental conditions were expected to be better able to buffer against environmental stresses. However, results of the present investigation show that neither of the above expectations was realised.

Rates of genetic gain in variable environments were similar to rates of gain in constant environments. In fact, rates of genetic gain in the cyclic and the random temperature lines were similar to the average of the rates of gain obtained in the three constant temperature selection lines. Furthermore, the rate of gain achieved in the random lines (R1, R2) was at least as large as that observed in any single line $\left(27^{\circ}, 24.5^{\circ}\right.$, or $\left.19^{\circ} \mathrm{C}\right)$ selected 
in a constant temperature. This outcome is to some extent anticipated by the high average genetic correlations between temperatures found in experiment one.

When selection lines raised under different environmental conditions were exposed to the three temperatures at generation 13 , highly significant genotype by environment interactions with respect to body weight were found in both variable and constant environments. Further analysis of the data revealed that the same interaction terms, while highly significant in all selected lines, were, except in one case, nonsignificant in the control lines. Clearly, the ability to resist environmental stress was altered by selection but not by the environmental regime.

There was a general decline in net fitness in lines selected for higher body weight in both variable and constant temperatures due to a loss in fecundity rather than a loss of fertility. This decrease could be due either to inbreeding in the selected lines or to the fact that individuals in the selected populations are phenotypically extreme individuals (Falconer, $1960 a$; Robertson, 1955, 1969). On the other hand, lines reared, but unselected, in these temperature regimes had fitness values comparable to those of the base populations. Additionally, different lines, reared under different selection pressures in different environments were qualitatively similar in their fitness responses to the test regimes, as indicated by the absence of significant genotype $\times$ environment interactions. Thus, in terms of fitness, lines evolved under constant environments appear to be no less flexible as compared with those evolved in variable environments.

Another measure of the ability of a particular line to cope with changing environments is its "buffering index" constructed here. Indexes of lines reared in constant environments are comparable to those of lines reared in variable environments in terms of body weight means. In fact, the lowest value, indicating the most uniform performance across testing temperatures, belongs to the line selected in the constant temperature of $24 \cdot 5^{\circ} \mathrm{C}$. Indexes for net fitness are higher for lines reared in variable environments due to low fecundity means in the $19^{\circ} \mathrm{C}$ temperature.

Our results, in agreement with the conclusion of earlier workers, show that genetic progress was greatest when the progeny were raised in the same environment in which the selection line evolved. However, the selection lines performed differently when progeny of each line were tested in all three temperatures. In particular, both control and selected lines reared at $24.5^{\circ} \mathrm{C}$ showed the highest fitness values both before and after selection as compared with flies reared in higher or lower temperatures. Additionally, the high body weight line selected under the $24.5^{\circ} \mathrm{C}$ temperature environment performed equally well in all other temperatures. If we assume that the optimum temperature constitutes a superior environment as compared with those represented by the other temperatures, then selection for overall performance should be carried out in the most favourable environment. This conclusion is in contradiction to those cited earlier. However, the environmental treatment used in the current experiment is different from those used in earlier experiments.

The results also show that lines evolved after many generations of exposure to variable temperature environments are not superior (in terms of ability to resist environmental changes) when compared with those reared in constant temperatures. However, these experiments were conducted 
over a period of only 13 generations. This is, of course, an extremely short length of time in terms of the history of populations and thus these results cannot be directly compared with those reported by Tantawy and Mallah (1960), for example. However, we can conclude that if organisms that have evolved through variable environments are more flexible, then this greater flexibility (or greater ability to resist environmental changes) probably was not acquired within 10-20 generations.

In view of the above, we concluded that long-term genetic gain in a quantitative trait through directional selection is probably unaffected in any major way by normal variations in environmental conditions and that selection is probably most efficient when the progeny are reared in the same environment as the selected parents. However, whether selection for performance in a variety of environments should be carried out under favourable or unfavourable environmental conditions is probably dependent upon the environment.

\section{REFERENCES}

ANDERSON, w. w. 1966. Genetic divergence in M. Vetukhiv's experimental populations of D. pseudoobscura 3. Divergence in body size. Genet. Res., 7, 225-266.

BAND, H. T. 1972. Further evidence of genetic and increasing developmental homeostasis in D. melanogaster during a minor climatic shift. Evolution, 26, 116-129.

BEARDMORE, J. A. 1960. Developmental stability in constant and fluctuating temperatures. Heredity, 14, 411-422.

BEARDMORE, J. AND LEVINE, L. 1963. Fitness and environmental variation 1. A study of some polymorphic populations of $D$. pseudoobscura. Evolution, 17, 121.

BROWN, J. H. AND FELDMETH, C. R. 1971. Evolution in constant and fluctuating temperature: Thermal tolerances of desert pupfish (Cyprinodon). Evolution, 25, 390-398.

DARLINGTON, C. D. 1939. The Evolution of Genetic Systems. Cambridge University Press, Cambridge.

DRUGER, M. 1962. Selection and body size in $D$. pseudoobscura at different temperatures. Genetics, 47, 209-222.

FALCONER, D. S. AND LATYSZEWSKI, M. 1952. The environment in relation to size in mice. J. Genetics, 52, 67-80.

FALCONER, D. S. 1960. Selection of mice for growth on high and low planes of nutrition. Genet. Res., 1, 91.

FAlCONER, D. S. 1960a. Introdution to Quantitative Genetics. The Ronald Press, New York.

FONTDEVILA, A. 1973. Genotype-temperature interaction in Drosophila melanogaster. II. Body weight. Genetics, 73, 125-134.

GOTTLIEB, F. J. AND SACKS, J. M. 1968. Fluctuating temperature and developmental stability. Genetics, 60, 183.

HARDIN, R. T. AND BELL, A. E. 1967. Two way selection for body weight in Tribolium on 2 levels of nutrition. Genet. Res., 9, 309-330.

HAZEL, L. N. 1943. The genetic basis for constructing selection indexes. Genetics, 28, 476-490. HILL, W. G. 1972. Estimation of realized heritabilities from selection experiments. II. Selection in one direction. Biometrics, 28, 767-780.

JINKS, J. L. AND CONNOLLY, v. 1973. Selection for specific and general response to environmental differences. Heredity, 30, 33-40.

JINKS, J. L. AND CONNOLLY, V. 1975. Determination of the environmental sensitivity of selection lines by the selection environment. Heredity, 34, 401-406.

LONG, T. 1970. Genetic effects of fluctuating temperatures in populations of D. melanogaster. Genetics, 66, 401-416.

MATHER, K. 1943. Polygenic inheritance and natural selection. Biol. Rev., 18, 32-64.

MACKAY, T. F. C. 1981. Genetic variation in varying environments. Genet. Res., 37, 79-93.

McDONALD, J. F. AND AYALA, F. J. 1974. Genetic response to environmental heterogeneity. Nature (Lond.), 250, 572-574.

NEVO, E. 1978. Genetic variation in natural populations: Patterns and theory. Theor. Pop. Biol., 13, 121-177. 
ROBERTSON, A. 1955. Selection in animals: Synthesis. CSH Symp. Quant. Biol., 20, 225-229. ROBERTSON, A. 1969. Proc. 12th Int. Cong. Genet., 3, 371-377.

TANTAWY, A. O. AND MALlAH, G. S. 1961. Studies on natural populations of Drosophila.

I. Heat resistance and geographical variation in $D$. melanogaster and $D$. simulans. Evolution, 15, 1-14.

THODAY, J. M. 1953. Components of fitness. Symp. SEB, 7, 96-113.

TURNER, H. N. AND YOUNG, S. S. Y. 1969. Quantitative Genetics in Sheep Breeding. Cornell Univ. Press, Ithaca. 